\title{
Screen printed and laminated electrodes for low-cost capacitive level measurement systems
}

\author{
Pavel Kulha ${ }^{* * *}$, Wolfgang Hilber ${ }^{*}$, Alexandr Laposa ${ }^{* *}$, Bernhard Jakoby $^{*}$
}

\begin{abstract}
The fabrication procedure and characterization of low-cost electrodes for capacitive level sensors realized on a flexible substrate are presented in this paper. The aim was to prepare conductive electrodes by printing of silver and PEDOT:PSS pastes on coated PET foil. Individual interdigital capacitors and a system with embedded microcontroller readout were designed for a comparative study. Individual capacitors in the form of interdigital electrodes (IDT) were designed with different finger width/spacing dimensions from $300 / 300 \mu \mathrm{m}$ to $800 / 800 \mu \mathrm{m}$, a finger length $10 \mathrm{~mm}$ and $15 \mathrm{~mm}$ and an overall length of $100 \mathrm{~mm}$. A demonstrator device featuring an integrated microcontroller, sensing and reference capacitive sensors and a resistive temperature sensor was realized to proof a practical utilization. The microcontroller is used to calculate capacitances of IDT electrodes in terms of charging time proportional to the fluid level. The design with reference capacitor can be directly applied to different fluids with a wide range of conductivities and dielectric constants without recalibration. The printed structures were thermally laminated with covering PET foil. The sensitivity of the fabricated devices was characterized in liquids with different relative permittivity and conductivity (water and oil). The highest measured sensitivity was $0.7 \mathrm{pF} / \mathrm{mm}$ and $0.08 \mathrm{pF} / \mathrm{mm}$ for water and oil respectively, with resolution down to $0.1 \mathrm{~mm}$.
\end{abstract}

K e y w or d s: level sensors, capacitance, printing, conductive polymer

\section{Introduction}

Polymer-based electronics are currently forming a new basis for low-cost microelectronic technology on typically thin, light-weight, and mechanically flexible substrates. Low-cost sensors are highly demanded, especially with the coming of the Internet of Things, and conventional microelectronic technologies usually do not meet the criteria regarding cost-effectiveness. The future in sensing technology will therefore supposedly partly lie in additive technologies of material printing using low-cost additive processes like screen, ink-jet or roll-to-roll printing on substrates such as plastic foils or paper [1]. Capacitive sensing has gained popularity in the detection of touch [2], force [3], chemicals [4], and level measurements [5, 6] due to the achievable sensitivity, accuracy, and easy processing directly with microcontrollers enabled for capacitance measurement. Capacitance level sensors can be used for wide variety of materials in a liquid or solid form (including powdered and granulated solids). The principle of operation is based on the fact that the air, which typically surrounds the insulated electrodes, is replaced by a material with a different relative permittivity [7].

The capacitive sensors for measurement of fluid levels have been heavily investigated and presented in a number of papers [2-12]. Different applications require different sensor topologies with respect to measured range, shape, flexibility, dimensions, fluid properties, the material of tank etc. Capacitive electrodes are mostly fabricated with cylindrical or coaxial $[8,9]$, parallel [10] or coplanar topology $[11,12]$, as is depicted in Fig. 1.

Capacitive level sensors are widely used in the manufacturing industry for liquid level detection in tanks or barrels, detection of fluid flow, and presence of granulated or pasty materials often in plastic or glass tubing. Highly

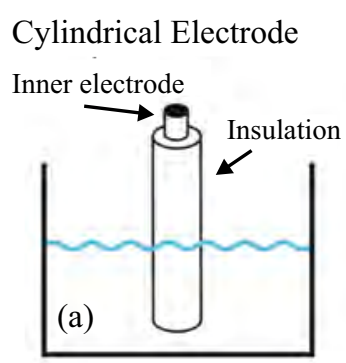

Ground electrode
Coaxial Electrodes

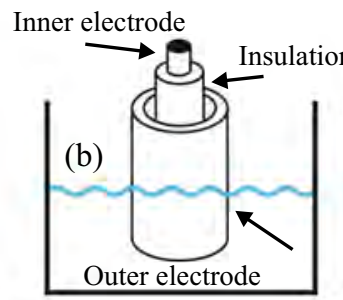

Non-concuctive tank

\section{Parallel Electrodes Co-planar Electrodes}

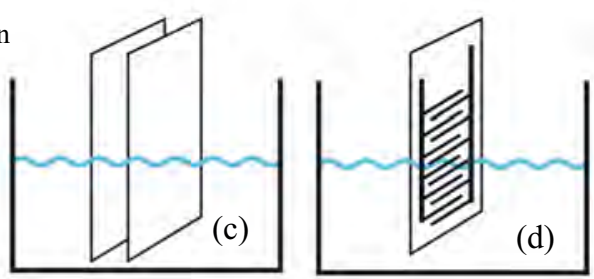

(d)

Fig. 1. : Basic topologies of capacitive sensors for fluid level measurement

* Institute for Microelectronics and Microsensors, Johannes Kepler University, Linz, Austria, wolfgang.hilber@jku.at, ** Department of Microelectronics, Faculty of Electrical Engineering, Czech Technical University in Prague, kulhap@fel.cvut.cz 


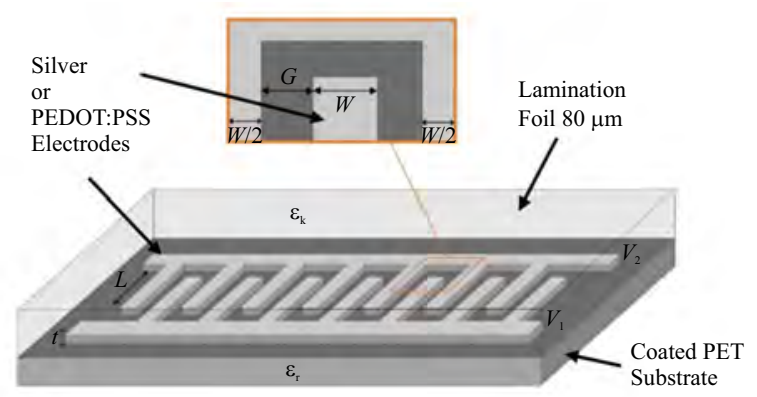

Fig. 2. : Structure of the interdigital capacitor (IDC)

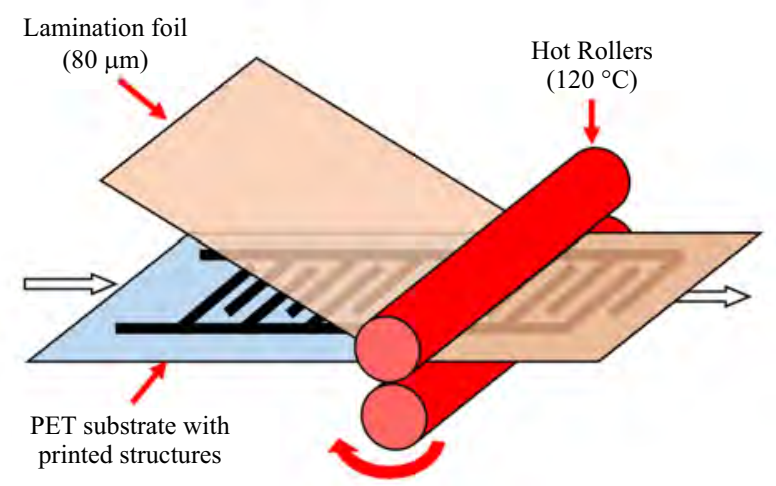

Fig. 3. Process of hot lamination

sensitive and flexible level sensors are currently demanded by the market.

\section{Materials, structures and methods}

\subsection{Sensor layout}

The comb drive or interdigital electrode capacitor (IDC, see Fig. 1) is probably the most used structure for level measurement due to its simplicity, versatility and easy planar manufacturing. Several footprints of IDC were designed with different finger width/spacing dimensions (W/G) from $300 / 300 \mu \mathrm{m}$ to $800 / 800 \mu \mathrm{m}$, finger length $(L) 10 \mathrm{~mm}$ and $15 \mathrm{~mm}$ and overall length of $90-100 \mathrm{~mm}$. Four individual sensors with scale were placed on the substrate with dimensions of $130 \times 70 \mathrm{~mm}^{2}$.

IDCs are suitable for level measurement of materials with a wide range of relative permittivity from $\varepsilon_{r}=1$ (air) to $\varepsilon_{r} \sim 80$ (water). Since the electrodes are insulated, the measurement of conductive liquids is also possible.

\subsection{Fabrication process}

The sensor structures were printed using the semiautomatic screen print machine RokuPrint SD05. A screen printing mesh with 120/34 threads/inch was used.

The printed electrodes were then thermally cured at the recommended temperatures for each ink. Silver paste (EDAG PF050 E\&C, Henkel, Düsseldorf, Germany) is cured at $121{ }^{\circ} \mathrm{C}$ for $15 \mathrm{~min}$. PEDOT:PSS (Heraeus Clevios ${ }^{\mathrm{TM}}$ ) paste is cured at $110^{\circ} \mathrm{C}$ for $5 \mathrm{~min}$. Thermal curing was done in a convection oven.

The microcontroller and passive components (a charging resistor for each capacitive sensor) are optionally placed using tweezers and connected using silver conductive paste (PF050).

The printed structures were contacted to the external terminal board using a silver adhesive and thin copper wires and then thermally laminated (at $120^{\circ} \mathrm{C}$, Fig. 3) with covering PET foil featuring a thickness of $80 \mu \mathrm{m}$. The lamination of the electrodes serves both, as a protection of the electrodes and a dielectric layer, and allows immersion and proper functionality in liquids being even highly conductive (eg, salt water). The laminated electrodes can also be stuck to the outer surface of nonconductive barrels or tanks. Moreover, PEDOT:PSS electrodes are semi-transparent as is depicted in Fig. 4.

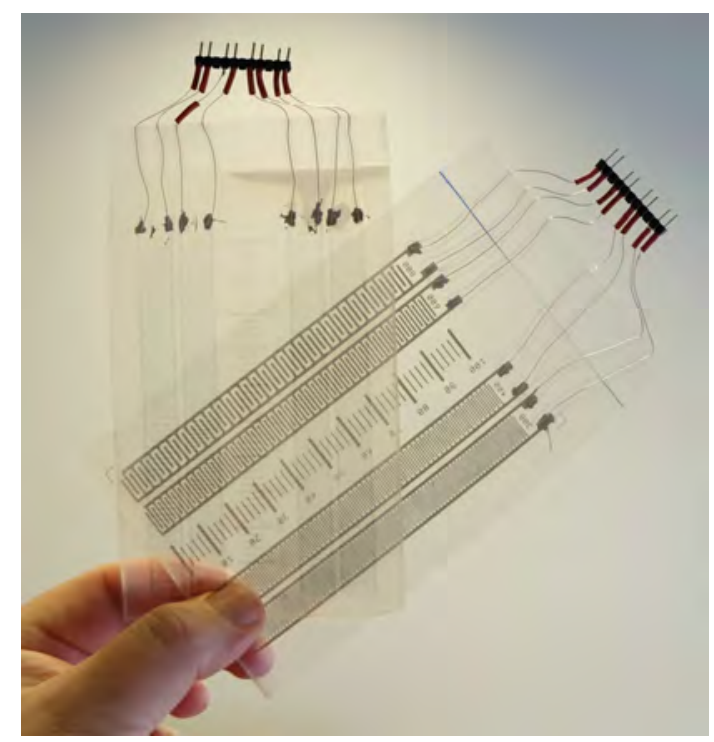

Fig. 4. Fabricated samples of four individual capacitive level sensors with silver and semi - transparent PEDOT:PSS electrodes (IDC finger length $10 \mathrm{~mm}$ )

The thickness and the 3D profile of the electrodes were scanned with a Bruker Dektak XT profile-meter. The thickness of the silver layer on PET substrate is $\sim 10 \mu \mathrm{m}$, the thickness of PEDOT:PSS is $\sim 300 \mathrm{~nm}$. The 3D scan of the IDT electrode is depicted in Fig. 5. The total thickness (including lamination) of the finished sensor is only $180 \mu \mathrm{m}$, and thus is highly flexible.

\subsection{Capacitance measurement using a microcontroller}

To keep the simplicity of manufacturing of the measuring system using screen printing, the measurement principle is based on measuring the $\mathrm{R}-\mathrm{C}$ time constant, since a minimum of external components beside microcontroller is required. It is commonly known that charging and discharging of a capacitor occurs exponentially with time, 


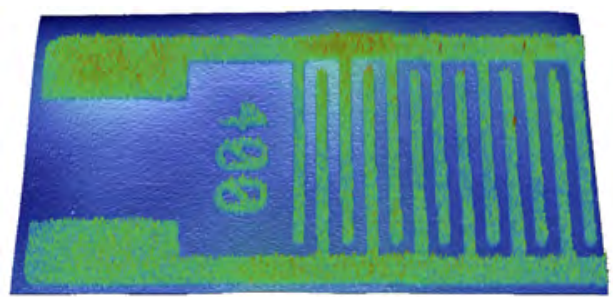

(a)

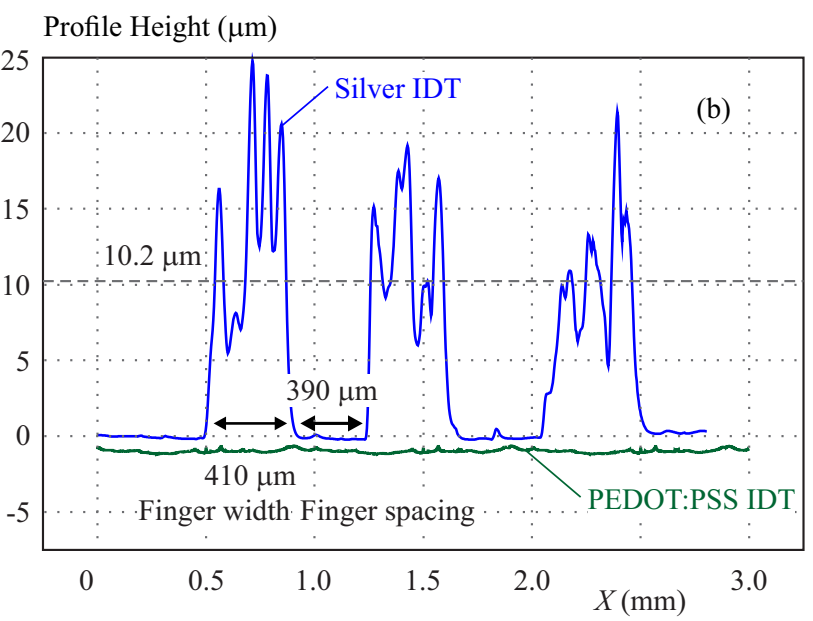

Fig. 5. 3D profile of silver interdigital electrode on pet substrate
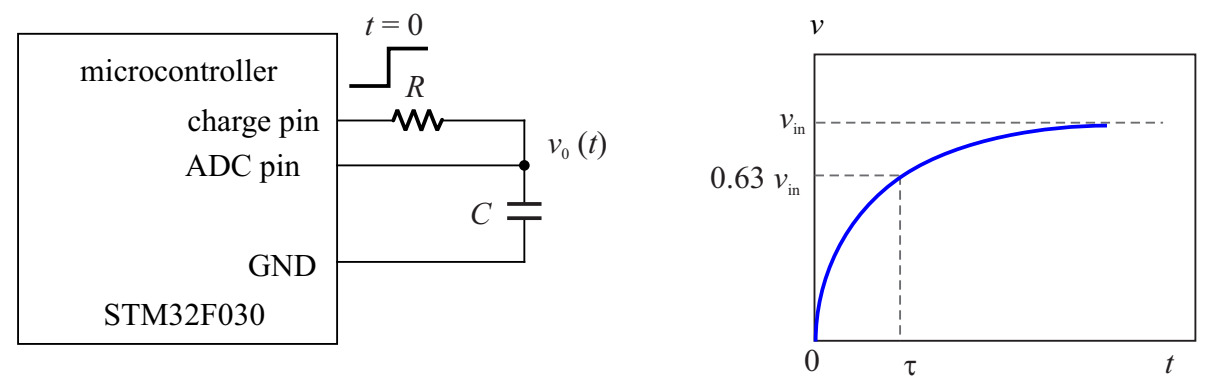

Fig. 6. Simplified schematic of the measurement procedure based on measuring the RC time constant

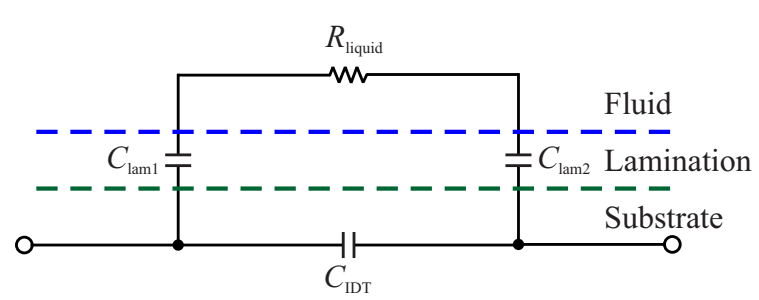

Fig. 7. Equivalent circuit of the laminated capacitive sensor

and the charging time depends on the resistance which is connected in series to the capacitor, see Fig. 6. When an uncharged capacitor $C$ is charged from a constant voltage source $V_{\text {in }}$ through a series resistor $R$ at time $t=0$ and $s$ - is the instantaneous voltage across the capacitor is given by

$$
V_{c a p}(t)=V_{i n}\left(1-e^{-\frac{t}{\tau}}\right)
$$

where $V_{\text {in }}$ is applied voltage and $\tau=R C$ is the time constant. In the time $t=\tau=R C$ we get

$$
V_{\text {cap }}(\tau)=0.63 V_{\text {in }}
$$

The microcontroller sets the charging pin to $3.3 \mathrm{~V}$ and starts the timer. An analog to digital converter assigned to the ADC pin (see Fig. 3.) continuously reads the instantaneous voltage across the capacitor until it reaches
$63 \%$ of $V_{i n}$ when the timer is stopped. From the measured charging time and the known resistance, we can calculate the capacitance.

An ARM-based STM32F030 microcontroller is used in the printed capacitive level measuring system. Only three external charging resistors are required for reading vertically and horizontally oriented sensing IDCs, reference IDCs and one resistor to form a half-bridge with a printed resistive temperature sensor. These sensors help to compensate the variation in the permittivity of the liquid measured (the reference capacitor must be fully immersed in the liquid during the measurement) and temperature changes.

As presented in [13], the electrical behavior of fluids depends on the excitation frequency. Water exhibits practically conductive behavior represented by an equivalent ohmic resistance at low-frequency operation (up to $\sim 100 \mathrm{kHz}$ ). The electrical characteristic (namely resistivity) is also affected by the concentration of dissolved solutes. The equivalent circuit of the laminated IDC structure is depicted in Fig. 7., where $\mathrm{C}_{\mathrm{IDT}}$ is the mutual capacitance of comb drive electrodes, $\mathrm{C}_{\mathrm{lam} 1,2}$ are capacitances of the laminated layer (which is influenced mainly by the thickness of the laminating foil), and $\mathrm{R}_{\text {liquid }}$ represents resistivity of the measured liquid. In the case of conductive liquids, increasing level of fluids gradually con- 

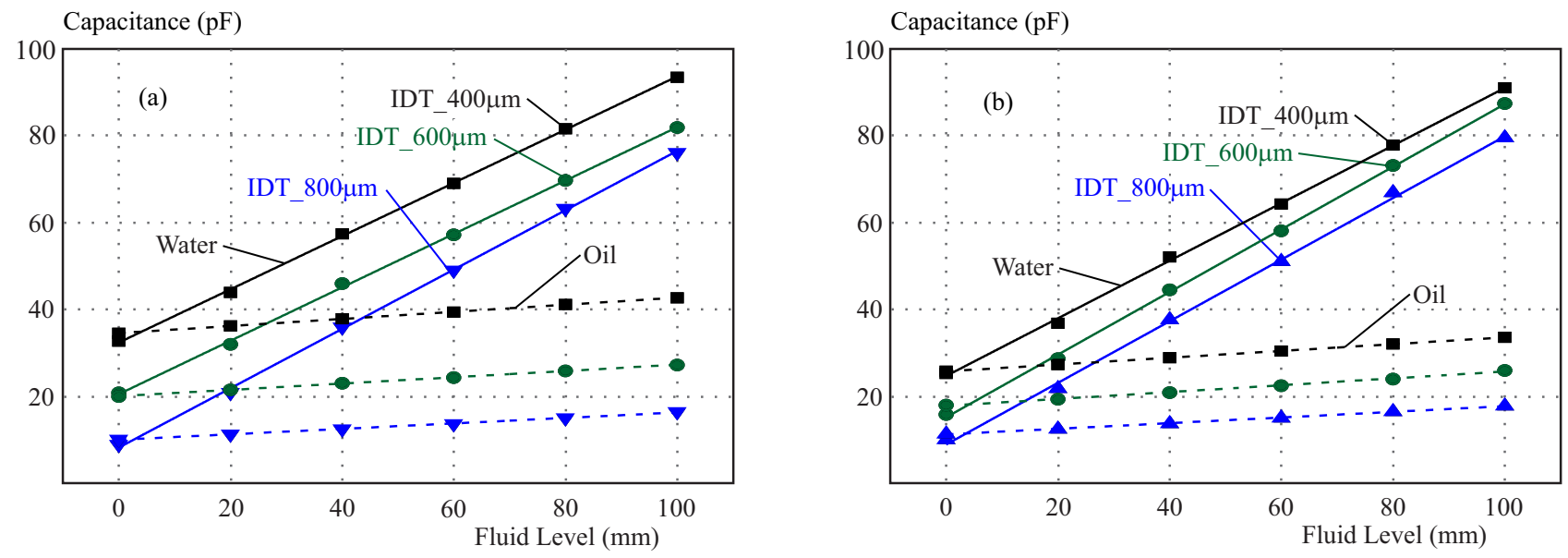

Fig. 8. (a) - Measurement results of silver electrodes, and (b) - PEDOT electrodes (right), showing an influence of the finger spacing and permittivity of the measured fluid
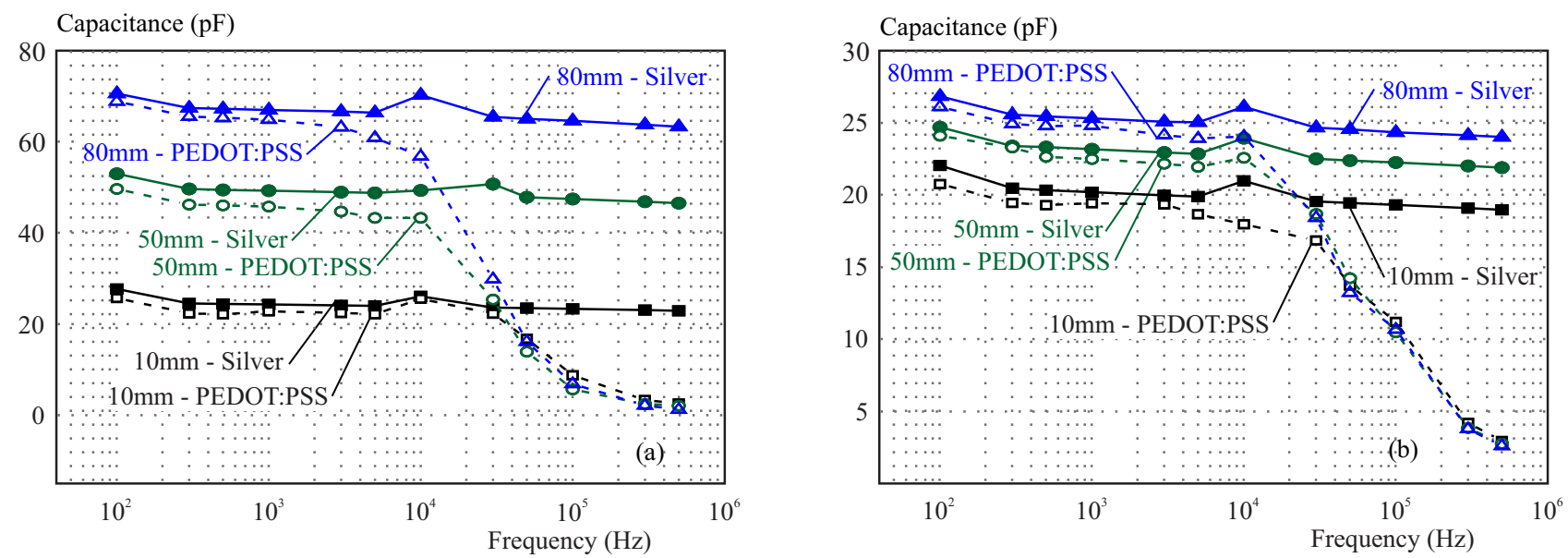

Fig. 9. Frequency characteristics of IDC with finger width/spacing $400 / 400 \mu \mathrm{m}$ : (a) - immersed in water, and (b) - immersed in oil

nects $\mathrm{C}_{\text {lam1 }}$ and $\mathrm{C}_{\text {lam2 }}$ in parallel to the $\mathrm{C}_{\mathrm{IDT}}$ and thus, the overall capacitance of the sensor increases.

\section{Measurement and results}

\subsection{Individual electrodes}

The laminated electrodes were placed in a plastic beaker which was filled gradually with a liquid under test (water, oil). The capacitance of the sensor was measured at each step by a precision RLC meter HP 4284A. The initial capacitance of printed sensor (without fluid in air environment) decreases with finger distance. The capacitance of printed sensor linearly increases with increasing level of the liquid in all cases (for different liquids and different IDT dimensions). The measured sensitivities (Fig. 8.) of the silver and the PEDOT:PSS electrodes are comparable. The sensitivity of the individual sensors to the water level is $0.61-0.68 \mathrm{pF} / \mathrm{mm}$ and $0.66-0.7 \mathrm{pF} / \mathrm{mm}$ for silver and PEDOT:PSS electrodes respectively. The sensitivity of the individual sensors to the oil level is $0.063-0.082 \mathrm{pF} / \mathrm{mm}$ and $0.034-0.061 \mathrm{pF} / \mathrm{mm}$ for silver and PEDOT:PSS electrodes respectively. The lower sensitivity measured for oil corresponds to its lower relative permittivity $\varepsilon_{\mathrm{r}, \text { oil }} \sim 3$. The results from measurements of frequency characteristics (Fig. 9) shows that the operating frequency should be kept in the range of units of kilohertz, especially for PEDOT:PSS electrodes.

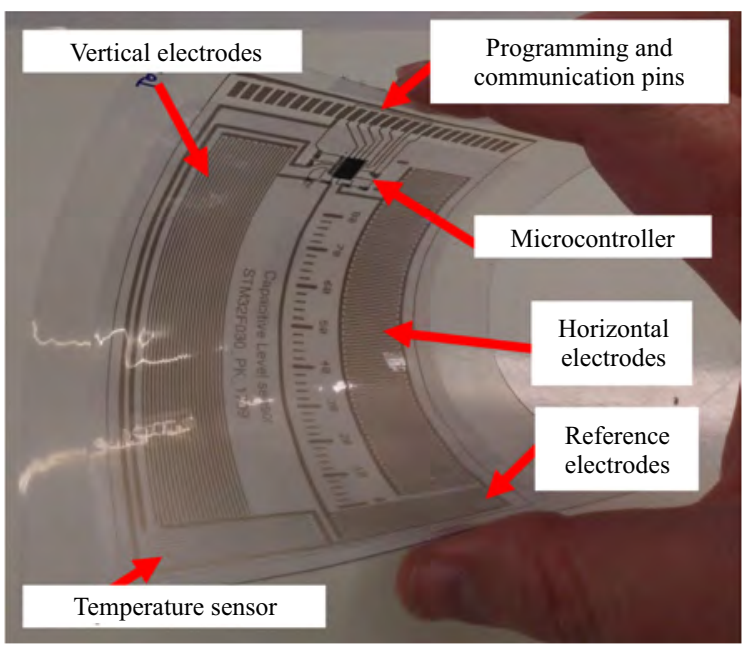

Fig. 10. Realized sample of the system with a microcontroller, horizontally and vertically oriented sensing electrodes, reference electrodes, and resistive temperature sensor 

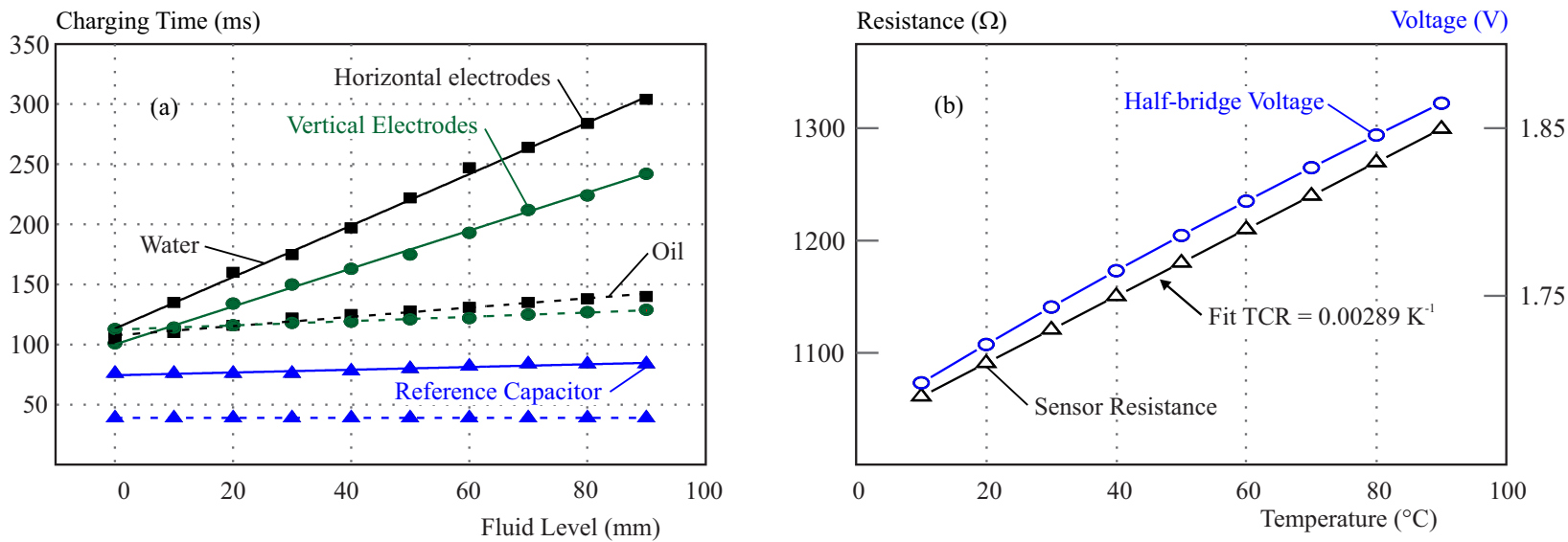

Fig. 11. (a)-charging time characteristics of horizontal, vertical and reference electrodes for water and oil, and (b) - temperature dependence of the printed resistive sensor and the half-bridge output voltage

\subsection{Demonstrator device with microcontroller}

As the next level of integration, a microcontroller capacitance meter based on an STM32F030 was designed and fabricated for a comparative study. The microcontroller in a tiny SMT package (TSSOP20) is integrated directly to the PET substrate close to the measuring electrodes. The realized sample of the printed flexible level measuring system and experimental results are depicted in Figs. 10 and 11. The sensitivity is higher compared to the IDC without microcontroller due to higher finger length $(15 \mathrm{~mm})$. The capacitance of reference electrodes is constant over the measured range and can be used for evaluating the permittivity of the measured fluids and measurement of the fluid level without recalibration.

The printed temperature sensors with $\mathrm{T}_{20}$ resistance of $1090.6 \Omega$ and calculated TCR $=0.00289 \mathrm{~K}^{-1}$ was connected in series with a $1 \mathrm{k} \Omega$ resistor to create a halfbridge. An output voltage of the half-bridge was measured directly by an internal 12-bit analog-to-digital converter (ADC) built in the microcontroller without a further conditioning. Just a small part of the ADC dynamic range is used to measure the temperature dependent voltage which leads to a relatively low temperature resolution of $0.4{ }^{\circ} \mathrm{C}$, but keeps the proposed measurement system extremely simple.

\section{Conclusions}

Screen printed and laminated electrodes for capacitive level sensors were successfully fabricated on flexible PET foil in the form of both individual interdigital capacitors and the integrated system with microcontroller readout. The fabricated structures were tested in liquids with different relative permittivity (water and oil).

The sensitivity of the individual sensors to the water level is $0.61-0.68 \mathrm{pF} / \mathrm{mm}$ and $0.66-0.7 \mathrm{pF} / \mathrm{mm}$ for silver and PEDOT:PSS electrodes respectively. The sensitivity of the individual sensors to the oil level is $0.063-0.082$
$\mathrm{pF} / \mathrm{mm}$ and $0.034-0.061 \mathrm{pF} / \mathrm{mm}$ for silver and PEDOT:PSS electrodes respectively. Characteristics of fabricated sensors are comparable with sensors fabricated on rigid substrates like a glass of FR4 [6] of flexible substrates like PET [11] or Kapton [5].

The integrated solution with a microcontroller measures the capacitor charging time in dependence on the level of the fluid with the highest sensitivity of $2.1 \mathrm{~ms} / \mathrm{mm}$. The main advantage of the system is that the measurement results can be sent to an acquisition unit in digital form using a serial bus (UART or $\mathrm{I}^{2} \mathrm{C}$ ). Moreover, the design contains also reference sensors for recognition of measured fluids (according to their relative permittivity) and temperature measurement. The resolution of temperature measurement is $0.4^{\circ} \mathrm{C}$.

The experimental results demonstrated that lamination of printed structures is a fast and low-cost method for a simple encapsulation and protection of printed layers and allows direct integration of conditioning circuits on the laminated substrate. This feature can also be used for integration of antennas with suitable RFID/NFC wireless functionality and fabrication of novel low-cost sensor nodes for the upcoming Internet of Things (IoT).

\section{Acknowledgements}

This work was supported by the Linz Center of Mechatronics (LCM) in the framework of the Austrian COMET-K2 programme and by the grant of CTU No. SGS17/136/OHK4/2T/13.

\section{REFERENCES}

[1] S. Logothetidis, "Handbook of Flexible Organic Electronics", Cambridge, UK, Woodhead Publishing, 2015.

[2] J. Sell, H. Enser, B. Jakoby, M. Schatzl-Linder, B. Strauss and W. Hilber, "Printed Embedded Transducers: Capacitive Touch Sensors Integrated into the Organic Coating of Metalic Substrates", IEEE Sensors Journal, vol. 16, no. 19, pp. 7101-7108, 2016. 
[3] M. Mentink, B. Van, Duren, D. Murray and H. Gill, "A Novel Flexible Capacitive Load Sensor for Use a Mobile Unicompartmental Knee Replacement Bearing: An Vitro Proof of Concept Study", Medical Engineering \& Physics, vol. 46, pp. 44-53, 2017.

[4] J. Hu, C. Yew, X. Chen, S. Feng, Q. Yang, S. Wang, W. Wee, B. Pingguan-Murphy, T. Lu and F. Xu, "Paper-Based Capacitive Sensors for Identification and Quantification of Chemicals at the Point of Care", Talanta, vol. 165, pp. 419-428, 2017.

[5] D. Paczesny, G. Tarapata, M. Michał and R. Jachowicz, "The Capacitive Sensor for Liquid Level Measurement Made with Ink-Jet Printing Technology", Procedia Engineering, vol. 120, pp. 731-735, 2015.

[6] S. Boonkirdram, "A Novel Planar Interdigital Capacitor Level Sensor", Przegląd Elektrotechniczny, vol. 1, no. 8, pp. 93-97, 2015.

[7] N. Zoric, A. Iavorschi, M. Sireteanu, G. Viziteu and R. Ciobanu, "Design and Simulations of IDC Sensor Using COMSOL Multiphysics and Dielectric Spectroscopy of LTCC Materials", Buletinul AGIR, vol. 3, pp. 63-69, 2013.

[8] K. Loizou and E. Koutroulis, "Water Level Sensing: State of the Art Review and Performance Evaluation of a Low-Cost Measurement System", Measurement, vol. 89, pp. 204-214, 2016.

[9] K. Loizou, E. Koutroulis, D. Zalikas and G. Liontas, "A Low-Cost Capacitive Sensor for Water Level Monitoring LargeScale Storage Tanks", 2015 IEEE International Conference on Industrial Technology (ICIT), 2015.

$[10]$ V. Bande, S. Pop, D. Pitica and I. Ciascai, "Electrical Model of a Capacitive Based Level Sensor", Proceedings of the 36th International Spring Seminar on Electronics Technology, 2013.

[11] M. Kisic, N. Blaz, C. Zlebic, L. Zivanov and M. Damnjanovic, "Flexible Inkjet Printed Sensor for Liquid Level Monitoring", 2015 38th International Spring Seminar on Electronics Technology (ISSE), 2015.

[12] K. Chetpattananondh, T. Tapoanoi, P. Phukpattaranont and N. Jindapetch, "A Self-Calibration Water Level Measurement using an Interdigital Capacitive Sensor", Sensors and Actuators A: Physical, vol. 209, pp. 175-182, 2014.

[13] F. Reverter, X. Li and G. Meijer, "Liquid-Level Measurement System based on a Remote Grounded Capacitive Sensor", Sensors and Actuators A: Physical, vol. 138, no. 1, pp. 1-8, 2007.

Received 21 December 2017

Pavel Kulha received his MSc degree in electronics from the Faculty of electrical engineering, Czech Technical University in Prague in 2002 and Ph.D. degree in electronics and microelectronics from the Faculty of electrical engineering, Czech Technical University in Prague in 2009. Since 2003 he has been an assistant professor at the Department of Microelectronics. From 2016 to 2017 he was a postdoc researcher at the Institute for Microelectronics and Microsensors, Johannes Kepler University Linz, Austria. His research interests include the design, modeling, development, and applications of microsystems and microsensors with a focus on embedded and printed sensors.

Wolfgang Hilber received the Dipl-Ing (MSc) degree in physics and the $\mathrm{PhD}$ degree in technical sciences from Johannes Kepler University (JKU), Linz, Austria, in 1993 and 1997, respectively. From 1994 to 1998, he was a Research Assistant with the Institute of Semiconductor and Solid State Physics, JKU. From 1998 to 2000, he collaborated in several research projects dealing with in-line process control for semiconductor manufacturing processes. In 2000, he joined the R\&D Division, E+E Electronics GmbH, Engerwitzdorf, Austria, where he conducted development projects in thin film sensor technology, mainly for automotive applications, until he finally joined the Institute for Microelectronics and Microsensors, JKU, in 2006, as an Assistant Professor. From 2017 he is an Associate professor at the same institute. His current research interests include microsensors and microfluidics.

Alexandr Laposa graduated in Automation and Computer Science from Faculty of Mechanical Engineering from the Brno University of Technology. Presently, he is working as a research fellow at the Department of Microelectronics, Czech Technical University in Prague, Czech Republic. Currently, he is working towards the $\mathrm{PhD}$ degree in piezoelectric resonators for gas sensing application.His work includes fabrication, simulation, measurement and data analysis of chemical sensors, electromechanical and thermomechanical modeling and simulation.

Bernhard Jakoby received the Dipl-Ing (MSc) degree in communication engineering and the $\mathrm{PhD}$ degree in electrical engineering from the Vienna University of Technology (VUT), Austria, in 1991 and 1994, respectively. In 2001, he obtained a Venia Legendi for Theoretical Electrical Engineering from VUT. From 1991 to 1994, he was a Research Assistant with the Institute of General Electrical Engineering and Electronics, VUT. Subsequently, he stayed as an Erwin Schrdinger Fellow with the University of Ghent, Belgium, performing research on the electrodynamics of complex media. From 1996 to 1999, he was a Research Associate and later an Assistant Professor with the Delft University of Technology, The Netherlands, working in the field of microacoustic sensors. From 1999 to 2001, he was with the Automotive Electronics Division, Robert Bosch GmbH, Germany, where he conducted development projects in the field of automotive liquid sensors. In 2001, he joined the newly formed Industrial Sensor Systems Group, VUT, as an Associate Professor. In 2005, he was appointed Full Professor of Microelectronics and Microsensors with Johannes Kepler University Linz, Austria. He is currently working in the field of liquid sensors and monitoring systems. 